\title{
Ethical implications of digital infrastructures for pluralistic perspectives
}

\author{
Maria Joseph Israel ${ }^{1} \cdot$ Ahmed Amer $^{1}$
}

Accepted: 19 January 2021 / Published online: 23 March 2021

(c) The Author(s) 2021

\begin{abstract}
It is important to design digital infrastructure that can better accommodate multicultural and pluralistic views from its foundations. It is insufficient to look at only the responses and influences of culture on technology without considering how the technology can be adapted in anticipation of, and to support, pluralistic multicultural perspectives in its original design. This goes beyond the simple act of supporting multiple languages and interfaces, but should include the ability of digital and data infrastructure to capture and accommodate pluralistic views, supporting multiple perspectives in the representation and processing of the data itself. In this work, we look at how rethinking the representation of data can allow us to more directly tackle domains that are typically hampered due to intercultural differences, and their inevitable losses in translation, particularly losses of valuable information like context and intention. When we refer to a loss of context and intention, we are referring to the loss of semantic information when practices such as referencing and citation are hard-coded to a particular set of cultural norms. We show that it is possible to expand the way in which we track referential data to capture richer semantic information regarding the contexts and intentions of the creators of this data, and thereby better serve the varied needs of those who consume, study, and refer to such data. We demonstrate this concept through a prototype system for a multicultural digital infrastructure, which we have named MultiVerse, and discuss its ethical implications from the perspectives of 'multistability', Intercultural Information Ethics framework, and poststructuralism.
\end{abstract}

Keywords Technology and ethics · Intercultural digital ethics · Ethical pluralism · Provenance $\cdot$ Data storage technology · Philosophy of technology

\section{Introduction}

As digital technology permeates everyday life, technology assessment researchers increasingly examine the embedded values in technology design and adaptation, and they do so from a variety of perspectives, including national, organizational, professional, and ethnic cultures (Watson et al. 1994; Gallivan and Srite 2005; Leidner 2006; Chilana et al. 2015). These technology assessments often take place after a technology is implemented and the ethical issues have become manifest. Instead of looking backwards on technology development, the technology analysis then gradually

Maria Joseph Israel

misrael@scu.edu

Ahmed Amer

aamer@scu.edu

1 Department of Computer Science and Engineering, Santa Clara University, Santa Clara, CA 95053, USA shifted focus to the technology design from the perspective of user-centered digital technology design principles and practices (Salvo 2001; Endsley 2016; Kushniruk and Nøhr 2016). Closely related to the user-centered technology design is the involvement of users and user data at every step of the technology design process, based on a number of user-centered design theories such as Participatory Design (Schuler et al. 1993; Spinuzzi 2005), Cooperative Design (Kyng and Greenbaum 1991), and Contextual Design (Beyer and Holtzblatt 1999; Holtzblatt 2009), besides others. A presupposition of these user-centered design theories and practices is the existence of direct correlation/connection between the design context and the use context. However, in reality, the underlying relation between technology design context and use context is more complex and unpredictable than what is presupposed by user-centered design theories (Albrechtslund 2007). In other words, every technology design faces the 'Collingridge' dilemma (Collingridge 1980). The Collingridge dilemma states that a technology 
in its early development phase can influence its development direction but not its impact on the society, and when the technology gets adapted into the society, it cannot alter its development. This dilemma emphasizes the dynamics of the interaction between technology design, development, and its usage.

Furthermore as argued by Barad (2007), it is not merely the interactions between humans and technologies that bring about the matter and meaning, but rather the intra-act. Here intra-act has to be understood, in the light of Lucy Suchman's work Human-Machine Reconfigurations: Plans and Situated Actions, in which she explains it thus: "whereas the construct of interaction suggests two entities, given in advance, that come together and engage in some kind of exchange, intraaction underscores the sense in which subjects and objects emerge through their encounters with each other" (Suchman 2007 , p. 267). From this perspective, the nature and meaning of technologies emerge from the intra-actions with other artifacts and with humans (van der Velden 2009). This implies that one has to go beyond mere ontological divisions and politics between humans and technology. Uncovering of the relational effects of technologies is important to adequately address the ethics of technology design and use in situations in which 'others' are concerned-such as other users, and users from cultures other than those of the designers, or users who were not anticipated during the design process - and doing so will help address "one's responsibility for the Other and the relationship between the Self and the unique, unknowable Other" (van der Velden 2009, p. 121) which is based on the Levinas' ethical philosophy of 'the Other' (Brigham and Introna 2007; van der Velden 2009, 2010).

Building on this perspective, we suggest shifting the focus from the consequences or impacts of technology, to the shaping and designing of technology with a specific focus on what data is preserved and how it is represented. We posit that technology must, by design, make provision for recorded communication being rich enough to avoid multicultural misunderstandings and problems wherever possible. We believe this can be approached by envisioning and adopting a richer data representation in the technology design phase. We argue that how data is preserved by technology is essentially a moral choice, and that what is preserved may directly or indirectly dictate how it is used. Moreover, a particular choice of data representation may imply the loss of certain data, such as intention, context, sentiment, or scope. In other words, there is a normative ethical assumption/decision being made, particularly in regard to multicultural communication, when a particular data representation is adopted. We argue that it is possible, and desirable, to develop multicultural infrastructure by design, and not just in the eventual operation of data systems after they are built. Our ethics and priorities can manifest in more than just how we manage our data once gathered, but in the structure and representation of the data itself.
To better understand how the structure of data representations can impact multicultural communication, and affect what information is lost or preserved, we need to consider how intercultural adaptation of existing technologies is lacking. For example, automated translation technologies ${ }^{1}$ may cause loss of data and accurate meaning when context and nuances of words are not taken into consideration. Similarly, 'like' or 'thumbs up' buttons or 'emoji' icons in social media technologies may not exactly capture users' intentions and emotions, thereby leading to loss of information, thereby potentially diminishing or warping the meaning behind users' behavior and their interaction with digital technologies.

Existing digital infrastructures take on a susceptive (an adapted reactive and active) role when it comes to the impact of (and on) cultural issues, but rarely discuss the potential to support pluralistic and varied views as a core part of the data infrastructure. In other words, existing adaptations to (and of) multicultural issues tend to presume an existing system that needs to be adapted, and not how a multiculturally sensitive system could be built from the ground up. Examples of systems that are adapted to multiple cultures would include current social media platforms, while examples of systems that attempt to adapt within an existing culture (or arguably to promote a particular culture, or to embody it), would include self-tracking systems. Obviously, these examples are limited and they function as representation of other cases and can be replaced by any other use case of digital technology design.

This paper will primarily discuss the subtle influence of how and what data are recorded and preserved, or how that has ethical implications on technology design and usage. We contend that our approach with MultiVerse ${ }^{2}$ is inherently

\footnotetext{
1 There are several audio, image, and video digital technologies. However, for brevity, we are restricting the discussion to texts and translation technologies, though the system can accommodate media of arbitrary format. These examples can therefore be easily replaced with any other digital technology for the discussion of multicultural perspectives and the technology design ethics.

2 The term "Multiverse" is widely used in different domains to describe different concepts. In science, it refers to everything that exists in totality (Carr and Ellis 2008) - as a hypothetical group of multiple universes. In quantum-computation, it refers to a reality in which many classical computations can occur simultaneously (Deutsch 2002). In a bibliographic-archival system, referred to as "Archival Multiverse", it denotes "the plurality of evidentiary texts (records in multiple forms and cultural contexts), memory-keeping practices and institutions, bureaucratic and personal motivations, community perspectives and needs, and cultural and legal constructs" (Archival Education and Research Institute (AERI) and Pluralizing the Archival Curriculum Group (PACG) 2011, p. 73). In Information Systems, it deals with the complexity, plurality, and increasingly postphysical nature of information flows (Gilliland and Willer 2014). Our
} 
better, as it tackles multicultural interactions and variances directly. We propose to leverage the existing ethical frameworks especially Ihde's phenomenological approach to technology known as 'multistability' (Ihde 1999, 2004, 2006, 2012), digital hermeneutics (Capurro 2010; Kelly 2016; Tripathi 2016; Romele et al. 2020), Intercultural Information Ethics framework (Capurro 2008, 2009; Hongladarom and Britz 2010), value sensitive design theory (Friedman et al. 2002, 2006) and hypertext/hypermedia of poststructuralism (Nelson 1965; Landow and Delany 1991; Landow 2006; Tredinnick 2007) to underscore the importance of supporting pluralistic views and interpretations which must start with the preservation of competing views and commentaries upon which they are based. In other words, it becomes essential to build an infrastructure capable of preserving and presenting multivariate views, and the transformational and referential processes that formed them.

The rest of this article is divided into the following parts. First, we present the prior work in the domain of technology design and ethical frameworks, as a background for technology design ethics. Secondly, current social media platforms and technologies aimed at shaping self and society will be discussed in order to establish that the structure and representation of data in technology design do manifest and shape ethical priorities and scenarios. Thirdly, the design of the MultiVerse project will be elaborated upon, to demonstrate the feasibility of designing a richer data representation in the technology design phase and of developing a multicultural infrastructure by design. Fourthly, the broader context and ethical implications of MultiVerse will be analyzed through the lens of digital hermeneutics, IIE, and poststructuralism. Finally, we conclude with a brief discussion on the broader application of MultiVerse.

\section{Background}

Attempts have been made to address the Collingridge dilemma either by anticipating ethical impacts of a technology known as 'technomoral scenarios/changes' (Swierstra et al. 2009) or regulating technological developments through 'sociotechnical experiments' (van de Poel 2013). Technomoral scenarios approach innovations as variations that are exposed to specific environments or markets, and attempt to speculate potential future impacts of a technology during their development. On the other hand, sociotechnical

\section{Footnote 2 (continued)}

use of the term "MultiVerse" with a capitalized 'V' denotes a version of our proposed digital infrastructure for a richer metadata representation, which captures the nature of representing multiple versions of a source data object, and was named partially due to the system's earliest tests being focused on translated poetry verses. experiments propose to accept uncertainty of future and approach innovations as social experiments that require responsible ethics considerations. Though these approaches recognize the relation between technology innovation and value change, as echoed by (Kudina and Verbeek 2019), they fall short of addressing the complex nature of technology design ethics, as the technomoral scenarios can only offer speculation about the future, and sociotechnical experiments can only allow for regulation without speculation.

This work relates to other prior works in the domain of technology design ethics including Ihde's phenomenological approach to technology dealing with the positivist's problem of designer's fallacy, Human Computer Interaction (HCI) and Value Sensitive Design theory (VSD), Intercultural Information Ethics (IIE), and poststructuralism, besides others.

\section{Multistability}

Ihde's concept of multistability emphasizes the fact that all technologies display ambiguous multistable possibilities and they can not be reduced to only their designed functions (Ihde 1999). This refers to the notion of 'multistability' which highlights the intrinsic nature of human-technology relationship which can acquire a basic meaning only in the context of technology usage and can take on a wide range of meanings depending on cultural, historical, political, and social contexts. Ihde uses Heidegger's hammer to argue the designed function of a hammer can not be limited only for driving a nail into an object, but rather it can be used for other purposes such as becoming an objet d'art, a murder weapon, a paperweight, etc. In other words, no technology is 'one thing', nor is it incapable of belonging to multiple contexts (Ihde 1999). Ihde claimed that there is a 'designer fallacy', which functions similarly to the 'intentional fallacy' in literature. That is to say that innovators cannot predetermine the use of their products, rather it is the users who determine how they use them, often without taking into account the inter-relations between the materials being worked with and their complex and multistable cultural contexts (Ihde 2006).

The problem of designer's fallacy relates to the inadequate recognition of the underlying complex and unpredictable future use contexts, as opposed to originally predicted use contexts, at design phase. Ihde's phenomenological approach to technology helps to abandon essentialist and substantivist understandings of technology design. We argue that this approach must be extended to information technology as well, in anticipating not only future use of technology features, but also anticipating context and intention of technology usage by capturing richer referential metadata, as is done in MultiVerse. 


\section{$\mathrm{HCl}$ and Value Sensitive Design}

In addition to the above, also relevant are developments in human computer interaction (HCI) and Value Sensitive Design Theory (VSD). HCI basically deals with the design of a system's functionality and usability (Preece et al. 1994; Karray 2008; Te'eni 2006), and to produce a three dimensional affective fit-between the user, the machine, and the required services-in order to achieve a certain performance both in quality and optimality of the services (Te'eni 2006). However, the HCI design paradigm moved through four evolutionary waves: its predominant focus of model-driven human factors, and technology tool functionalities in its first wave; to user-centered work contexts and efficient interactions within well established communities of practice based on participatory design theories in its second wave; and on to broader use contexts, affective interactions, embodied actions, and situated aspects of user activities in its third wave (Preece et al. 1994; Bødker 2006,2015; Karray et al. 2008; Fernaeus et al. 2009; Helander 2014); most recently moving to 'politics, values, and ethics' in its emerging fourth wave (Ashby et al. 2019). Our richer metadata design for representing user embodied actions relates to the current research on HCI, in particular its third and fourth waves.

VSD, as stated by Friedman et al., is "a theoretically grounded approach to the design of technology that accounts for human values in a principled and comprehensive manner throughout the design process" (2002, p. 1). It consists of an iterative tripartite methodology of conceptual, empirical, and technical investigations to analyze embedded human values throughout the design process. Building on the theories and methods of Computer Ethics, Social Informatics, Computer Supported Cooperative Work, and Participatory Design, it presents 'a unique constellation of features' including the following: being proactive in the entire design process, examining enlarged arena of workplace and non-workplace, broader scope of human values with moral import, utilizing the iterative tripartite methodology of conceptual, empirical and technical investigations, focusing on dynamic interactional aspects of technology, people, and society, drawing on moral epistemology, and recognizing universally held values in specific use contexts (Friedman et al. 2002, 2006).

One of the key positions of VSD is that technology in general has a disposition for either supporting or diminishing certain values by a particular technology design, as seen in the previous example of a hammer. While the technical investigation shapes technology based on values identified in the conceptual analysis, the empirical analysis focuses on the human context in which the technical artifact is situated and activity that can be observed, measured, or documented. It is this dimension of technology our MultiVerse infrastructure aims, in an explicit way with richer metadata, to capture user behavior, context, and sentiments, and map inherent values emerging from people and social systems influenced by a technology artifact.

There are other relevant works such as digital hermeneutics, IIE, poststructuralism-hypertext/hypermedia which are discussed below (under "Broader context and ethical implications").

\section{Digital infrastructure and current social media platforms}

Let us examine briefly how current digital infrastructures of social media are designed and utilized, and how they affect the loss or preservation of multicultural communication. As an example, let us imagine that Alice posts a news story about refugees and then her online acquaintance, Bob, gets upsets and clicks a metaphorical dislike button by, e.g. posting an angry emoji. Now we can raise the question: What is Bob angry about? Is he upset about the plight of the refugees or about a cruel comment Alice made about refugees in her social media posts? Emotions of users, and the context of their social media posts, are not accurately captured by existing social media platforms. Now imagine if Alice's post had originally been written in a language that Bob did not natively read. Instead, Bob was reacting to an automatic translation of Alice's original comments on the shared posts. Is Bob's angry reaction a response to the post as intended, to the content to which it refers, or to something lost (or added) in translation?

It is obvious that the effects of digital technology and the internet on translation is continuous and widespread. Moreover, the potential instantaneity and accessibility of digital media imply a greater access and acceleration of automated translations with potentially disruptive effects. The widespread dissemination of translations can depict other politics and cultures to the dominant political, economic, and cultural agendas (Cronin 2012). One such example is the use of social media during the Egyptian Revolution in 2011. The Arab Spring was significant and has been widely commented upon. It is notable that when analysts began to consider the implications of what was happening during the Arab Spring, they began to turn to translation as a framework of explanation (Mehrez 2012). There is no doubt that the Arab Spring movement, mediated by social media and translations, enabled massive protests. But the translated texts lacked clarity on Egyptian cultural contexts, religious elements, and pan-Arab issues. Particularly, it failed to capture the precise contexts, tone, and sentiments of the public due to the inherent technology design flaws as discussed at the beginning of this section. Such technology design drawbacks further complicated the meaning and interpretation of the reality, and eventually exasperated the movement. 
At another level, the imprecise translation flaws of the digital new order also impact migrants beyond one's imagination. The realities of millions of migrants are very vivid, especially their continuous struggle to cross geographical borders in search of better livelihoods using mobile devices and other internet technologies to navigate unknown cultural and language barriers. In this process, they are often caught up in "that translation zone between life in a new language and continued digital interactivity with the language in their country of origin" (Cronin 2012, p. 8).

Therefore, it is evident that the interconnection of social media applications as an ecosystem may seem to present and preserve complex interactions and content, but when we consider translation, it can easily lose intent, tone, and context. This can result in a distorted image being conveyed between cultures, exasperating cross-cultural communication problems, and seems to be more than merely a side-effect of crossing a linguistic barrier, but involves the interplay of technology. We consider the implications of large-scale social media platforms, and then how our proposed MultiVerse system offers an approach to tackling the distorted translation problem.

\section{Technologies aimed at shaping self and society}

Another relevant topic is technologies that draw on big data to nudge individuals with personalized feedback to change behavior known as 'hypernudging' (Yeung 2017; Lanzing 2019), either for the reshaping of an individual or a society. These personal hypernudging technologies, include self-tracking technologies either by an individual or by an external party. Personal self-tracking technologies refer to both wearable devices and software applications. These technologies provide a mechanism to explore personal goals, project complexity, and technological involvement in one's life (Butterfield 2012). These technologies may also provide mechanisms to enhance one's performance in terms of self-healing, self-discipline, self-design, self-association, or self-entertainment (Gimpel et al. 2013). However, research on self-tracking technologies, also known as 'quantified self technologies', is primarily done to explore personal and social meanings of self-technologies in their present form as they are (after-the-fact).

An example of a large scale self-tracking scheme by an external party is the Social Credit System (社会信用体系 shehui xinyong tixi), (SCS), in China (Botsman 2017; Lanzing 2019; Kostka 2019; Wong and Dobson 2019). The SCS is an online system of benefits and sanctions that aims to steer the behavior of people and institutions and thus rates the trustworthiness of 1.3 billion Chinese citizens (Botsman 2017; Lanzing 2019; Wong and Dobson 2019). The SCS system, such as the Sesame Credit developed by Ant Financial Group, collects massive volumes of data on people's online activities such as their purchases, bill payments, posted content on social media, interaction with friends, video games, etc., and evaluates them either positive or negative, and assigns a citizenship score according to rules set by the government. Based on this quantified score, they are ranked against the entire population of China, which can determine their eligibility for certain benefits, like a mortgage or a job or their children's school admission. This citizenship score is not just an indication of their social behavior, but is also designed to nudge them towards an 'acceptable' behavior approved by the government, thereby promoting a nationwide sense of trust and sincerity. While one might expect that people, across different age groups, may be concerned about privacy implications of SCS, they instead appear to embrace SCS with high levels of approval (80\%), according to a cross-regional survey by Kostka (2019). In contrast to previous research studies that perceived SCS as a surveillance infrastructure for social management (Creemers 2018; Hoffman 2017; Liang et al. 2018; Wong and Dobson 2019), the survey further reveals factors that influence people to embrace the SCS include: individual's characteristics such as demographic factors, online presence, and political attitude; SCS' characteristics in terms of its score magnitude, transparency, and fairness; perceived and received advantages and disadvantages of SCS such as improved accountability, quality of life, and conformance to government regulations. In particular, the rationale for such a positive response to SCS is because they view SCS as a system promoting "honest dealings in society and economy instead of privacy-violations" (Kostka 2019, p. 1566).

In other words, behavioral responses to SCS are driven mainly by material or symbolic (i.e. social recognition) motivations to influence scores in a positive way rather than punitive elements playing a role, which could also influenced by (linked to) the Confucian notion of self and identity as relational based on the five cardinal relationships: between ruler and minister, between father and son, between husband and wife, between brothers, and between friends. In Chinese traditions deeply grounded in and shaped by confucian thought and ethics, the self is what Ho (1993) calls the relational self, one which is intensely aware of the social presence of other human beings. Such relational nature of selfhood, that is to say, self is subdued and constricted to respond to perceptions of social requirements and obligations, as stated in Analects (Conversation of Confucius), "To subdue one's self and return to propriety is perfect virtue" [XII. 1. (Soothill 1910 p. 557)], where 'propriety' refers to prescriptive rules for proper conduct and sincerity to unwavering devotion to the good (Ho 1995). Therefore, from this perspective of the selfhood in Confucian cultures forming internal psychological dispositions and the present external authoritarian socio-political institutions, adherence to the SCS norms seem plausible, as the Kostka's survey study 
notes. Obviously, self-cultivation in Confucian thought is clearly different from the notions of self-actualization held by many humanistic psychologists and philosophers in the West. It should be noted however, that more recent western ideas may be more in line with aspects of such a positive look at social credit's non-punitive appeal. For example, Thomas M. Scanlon's idea of interpersonal morality, described as 'contractualism' in his book What We Owe To Each Other, giving others their due is nothing but involves being able to justify one's conduct to others (Scanlon 2000; Watson 2002). This implies that doing right by other people is nothing but treating them in ways they cannot reasonably reject.

In contrast, a critical analysis of the design of the Chinese SCS, i.e. the set of mechanisms providing rewards or punishments as feedback to actors for the morality of their socio-economic and political actions (Lanzing 2019), candidly critiqued it as an 'Orwellian nightmare' (Orgad and Reijers 2019), and as a big data gamified version of modern social control/management (Creemers 2018; Hoffman 2017) and state surveillance (Liang et al. 2018; Wong and Dobson 2019). To address these issues, it is not sufficient to enable a system to work at a technical level, not least because the quality of the data that goes into the system itself may be suspect, compromising the reliability of its outcomes. Moreover, a particular problem will arise when individuals try to game the system by artificially maximizing particular metrics. Compatibility and interoperability need to be ensured between data storage formats and central and local systems, operated by both public and private actors. Subsequently, the data needs to be processed and interpreted in a way that ensures the accurate construal of meaning from the data results, if there is to be any reasonable hope of translating such data into useful information and legitimate decisions.

Naive self-tracking technologies and SCS proposals, may assume that the information technology gathers all users' information accurately. In fact, these technologies are not designed to capture richer metadata like personal contexts, and social contexts of people's online activities and their corresponding frequencies. An online exchange between an individual and an extremist may be lengthy due to their agreement, or disagreement, on the issues they are debating. Simply concluding that such two parties interacted heavily would inevitably lose such a critical context. This is an example of what we term 'richer' metadata that could have been captured had a system been designed with such an ability in mind from the beginning. These losses of richer metadata can be permanent, unless there is a way to preserve the original form and translated forms of media, and adding commentaries such that they too can be preserved. Without this, they might never be interrogated and examined should they be relevant to making, or reviewing, a critical decision. In other words, it is only helpful when as much context as possible is recorded. This could include at a possible minimum, the context, scope, and intended tone of the original content.

To this end, we have developed and experimented with interfaces and data representations to maintain such diverse and multivariate media. One initial prototype, that we describe next, is known as MultiVerse (which shows how multiple translations of poetic verse can be preserved in a single multivariate repository that allows interrogation and tracking of how any particular derived translation was formed).

\section{MultiVerse}

We, in the first part of this section, briefly explain the nutsand-bolts and in the second part, consider the essential design principles and ethical benefits of, MultiVerse project. MultiVerse is designed as a digital data infrastructure that preserves multiple perspectives, and thereby allows better support for multicultural digital content. We contend that in order to better support intercultural ethics, and more ethical digital media curation across cultures, such an infrastructure is needed. So, what is MultiVerse? MultiVerse is a digital data representation infrastructure intended to track provenance of multi-varied translations of scholarly texts and their derivatives. MultiVerse infrastructure allows users to remix existing translations and/or add one's own personal translations at will. Provenance can be defined as the recording of the history of user activities that create and transform data. MultiVerse is primarily concerned with the metadata needed to store such provenance alongside the data to which it refers. In this project, provenance tracking is done by capturing all translations (users' activities) without any preferences, prejudices, or prizes (value judgements/ correctness), at the time of their composition.

To realize this concept, we have used the well known thirteenth century Italian poet Dante Alighieri's the Divine Comedy, and some of its many English translations (The Dartmouth Dante Project 2013). We have combined these into a single repository that allows the remixing and composition of new translations, while offering detailed tracking of the origins and transformations of such texts. A user has the option of either collating different versions of verses or adding in his/her versions of verses from/to this repository to compose his/her unique version of translation of the Divine Comedy. Moreover, the user can explicitly tag richer semantic metadata like context, intent, scope, or tone/sentiment to his/her composition. Multiple versions of the Divine Comedy are thereby stored in a single repository with rich version histories. To keep such a repository consistent, it is structured as an immutable data store, allowing the addition of new content and amendments, but disallowing any modification or deletion of data that has been committed to 


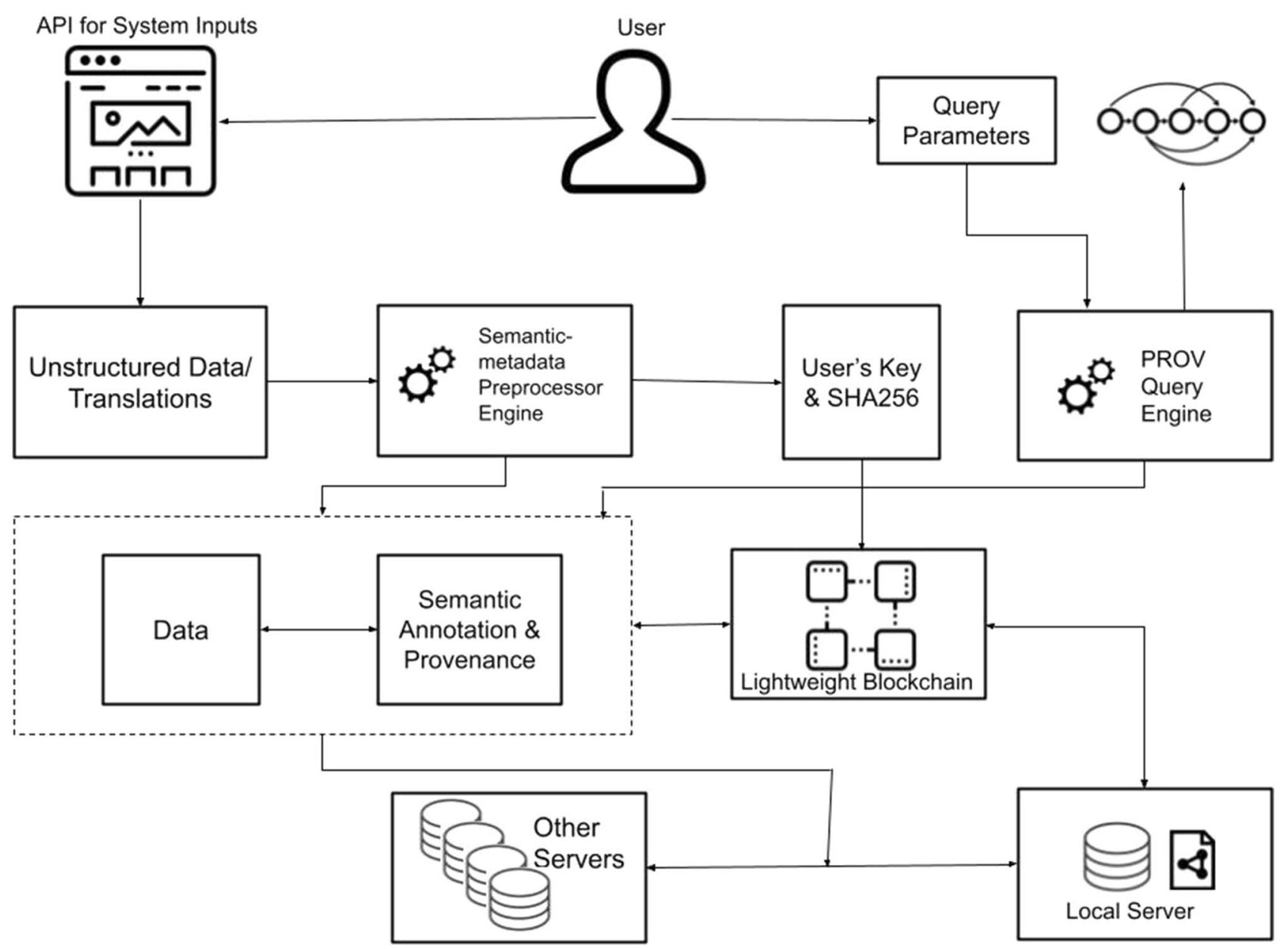

Fig. 1 MultiVerse's architecture overview

this store. The immutable aspect of MultiVerse is achieved by adapting a basic model of blockchain technology, without worrying about cryptocurrencies. The interactive aspects of MultiVerse are enabled by offering a user application programming interface (API) to allow access to the repository. A high level architectural overview, the user API of MultiVerse, and a sample query result, are depicted in Figs. 1, 2, and 3 respectively.

The primary purpose of this project is to demonstrate the importance of capturing and representing pluralistic views cutting across individuals' cultural, ethnic, religious, gender, social, political, economic, emotional, etc. stances/ viewpoints. At its very beginning, a key design principle of MultiVerse is to enhance technology to represent pluralistic multicultural perspectives of all users, rather than after-thefact. This is achieved by designing MultiVerse which enables users to record not only their views irrespective of their correctness but also explicitly accommodate their contexts and intents.
We might ask, "what are the benefits of this technology design principle in the first place?" Without arguments, it can be stated that all voices are preserved. Single versions can be presented on demand. This might appear as if all that we do is record all voices, but that would be inaccurate, as the most basic support for pluralism requires recording both the multiple voices, as well as sufficient information to present their relationships and contexts. This is necessary for the most basic forms of multicultural pluralism, and yet it can be built upon for more complex and richer solutions. We discuss pluralism, and especially interpretive pluralism below (under "Broader context and ethical implications") (and in particular ("IIE and interpretive pluralism")). Further, the history and identity of those who created or selected the individual versions, and the provenance of such documents would need to be permanently stored, and protected from any tampering. For that reason, MultiVerse implements an archival data store that is designed to be immutable. And by virtue of its immutability, MultiVerse becomes a means to establish the source of any loss of nuance, and makes 


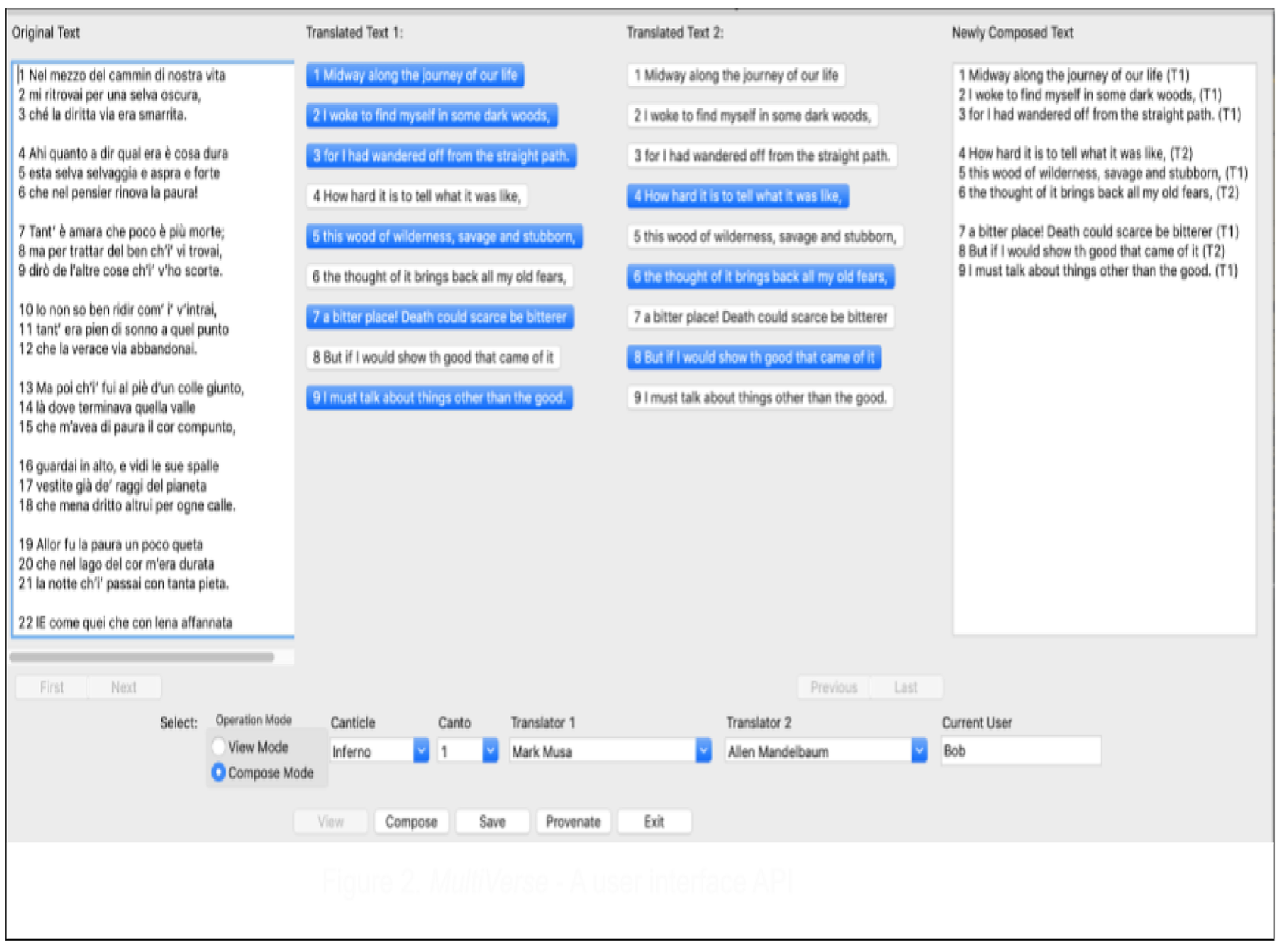

Fig. 2 MultiVerse-A user interface API

arguments (by allowing future archaeology on such repositories) about the correct form moot. More precisely, while it does not eliminate contention over the ideal translation, it does not force that debate to be fought over the preserved version. There need be no permanent winner, and past mistakes can be corrected in future revisions. But this leads us to consider the broader ethical implications of such multicultural pluralistic digital infrastructures. Ethical implications of MultiVerse framework are discussed in the next section.

\section{Broader context and ethical implications}

Creating a richer representation of data, its context, and its provenance has ethical implications, and a broad range of potential benefits. In particular, it offers a means of supporting multicultural perspectives through the data storage infrastructure itself, rather than imposing the traditional demand that adapted or translated data be presented without the contexts and provenance links that help reconstruct its path to creation. Such additional metadata has a direct benefit in removing contention between alternative interpretations seeking to become the de facto canonical view in a particular cultural perspective. In this section, we present the broader context/relevance of MultiVerse discussing digital hermeneutics, IIE, and poststructuralism.

\section{Digital hermeneutics and contextualism}

The problems we are experiencing in social media platforms and self-tacking technologies_-personal and public-are a microcosm of a broader technological culture in which we live. The philosophers of technologies attempt to comprehend the present technological culture by developing a 'praxis philosophy of technologies' (Tripathi 2016). The role of philosophy of technology is to uncover the hidden or unconscious cultural and social expectations/orientations of designers and users (Kelly 2016). To this end, hermeneutics, as a tool within the philosophy of technology, aims to 


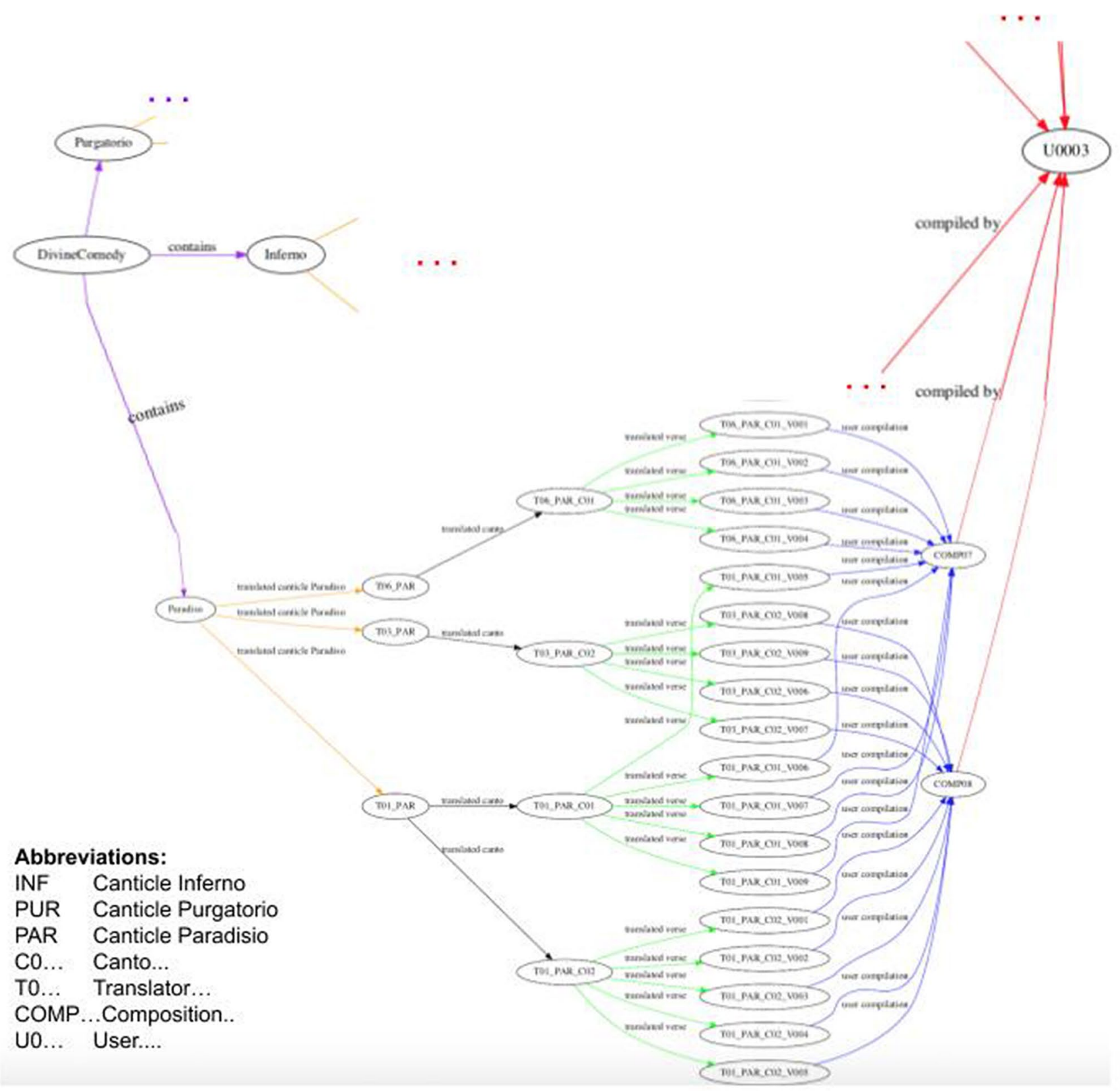

Fig. 3 A section of MultiVerse query result representing user composition contexts

discover the primary meaning of a text as intended by its author. Traditional hermeneutics has thereby been extended as a material hermeneutics, to understand the material culture and technologies. Furthermore, a newer approach, known as digital hermeneutics, has been proposed to evaluate the ethics of the information technological culture of everyday life, such as the burden of information overload, and the increasing ubiquity of the internet, social media, and cyberspace (Capurro 2010). Digital hermeneutics lies at the cross junction of material hermeneutics and digital technologies that produce digital artifacts/texts, and try to subject them to meaningful interpretations (Capurro 2010; Tripathi
2016; Romele et al. 2020). The aim of digital hermeneutics, in line with that of the material hermeneutics, is the process of understanding the praxis of information technologies that are culturally and socially embedded (Tripathi 2016). We appeal to this method of evaluation in understanding the technology design and ethics of our approach to supporting multi-cultural infrastructure. In particular, we discuss MultiVerse as an exemplar of technology adaptation and use, which allows us to evaluate it from the very beginning of its planning stage.

Scholars agree that all understanding/knowledge is context dependent/sensitive (Stine 1976; Cohen 1999), 
often within multiple rings of context. The contexts can be divided into the immediate, broader, and remote contexts. The immediate context refers to a given piece of passage which contains immediate references within the given words, phrases, clauses, sentences, paragraphs, or sections. Some helpful tools to comprehend the immediate context are evidently language tools like vocabulary, syntax, and grammar. The broader context places a given passage within the overall scheme of a literary work as a whole and/or other works by the same author or a particular genre, while the remote context deals with the even wider context of the passage in history and its historical circumstances-time, place, and purpose.

As these different contexts are important in interpreting the technology design and use, MultiVerse captures these contexts in the use case of Dante's popular literary work, The Divine Comedy (DC). In the DC, the immediate contexts are particular verses, cantos or canticles, while the broader contexts are the relationships between verses, cantos, and canticles in the form of their relationships to each other, their scope, and the intended tone within the overall DC as a literary work. Specifically the referential relationships that define context in this case can include: "part_of", "consists_of", "translated_copy_of", and "composed_copy_of." The remote context deals with the historical, cultural, and social contexts of the written passages and their derivatives.

We believe that since context can provide information about recorded data, it should be, to the best of our ability, made manifest in the form of data. This means that we need to look a little more deeply at what context can potentially mean, so as to understand why a broad and flexible approach to gathering and representing additional context data can be crucial in better communicating and supporting the preservation of data in a multicultural world.

Critics of contextualism would critique that contextualists argue for their view on knowledge not by making epistemological claims but primarily by making careful observations about how we use the word 'know' in ordinary life (Cappelen and Lepore 2005). If the contextualists are right that there is no general univocal question about what we can or can not know, then there are only questions we can accurately describe ourselves as knowing in different contexts. Knowing by ordinary standards relative to ordinary alternatives is enough for ordinary purposes. A skeptic is right that we do not know by his own hyper-inflated standards. But according to contextualists, to base the case for skepticism on this observation is to fall victim to a trick of language (Stine 1976; Cohen 1999). This would suggest that to truly capture knowledge and the lack thereof, we need to have somehow preserved the context of the data that has been recorded. This in turn makes it essential to understand the pitfalls of attempting to capture data under the presumption that there is a single correct context. We therefore invoke the designer's fallacy to illustrate the need to be explicit about the recording of intent. By allowing for the preservation of metadata that can be used to indicate not simply the provenance of documents, but which allows for the indication (and preservation) or declared or implied intent in the creation of a document, MultiVerse, attempts to provide a means of explicitly recording individual and varied contexts-multivariate interpretation of texts.

\section{Multistability and multiculturality (multicultural views)}

Another concern with a system like MultiVerse is the positivist problem of presupposing the connection between the design context and the use context. In fact the relation between design and use is very complex and unpredictable (Albrechtslund 2007). It is indeed important to be open to future use contexts, and we would argue that a system like MultiVerse attempts to anticipate this problem by gathering metadata that is fundamentally relational in nature, but which also includes clear tracking of authorship and origins of the added metadata. This does not eliminate the problem entirely, but it does shift the burden of evaluating any bias from the building of the system, by allowing deferred evaluation of origins and authors. This means that while a potential future use may indeed be implied by a particular contributor of metadata to the system, this can be taken into consideration for future evaluations wherever possible. This kind of flexibility may be better understood through the lens of Don Ihde's concept of multistability.

Don Ihde's multistability is a phenomenological approach which states that the use of a technology is not restricted to its original design because its usage can go beyond its original design goal (Ihde 2004). Ihde's concept of multistability highlights the possible wide range of purposes that can be conceived differently according to cultural, historical, political, and social contexts (Ihde 2004, 2006, 2012). For example, an ordinary screwdriver might not serve well as a ladle, pillow or wheel, but it can certainly be used for many other purposes than tightening screws. It can be used in contexts that we can hardly imagine, such as a tent-peg, as a hammer to hammer down such a peg, or as a tool for artists to scratch their artwork onto a suitable surface. For a system like MultiVerse, cultivating such multistability is in fact a goal. We aim to ensure, as much as possible, that no presuppositions are built into the design regarding how the data might be useful in future. This is important for a system like MultiVerse, that aims to preserve information, so that it might be useful in as broad a set of domains as we might currently contemplate, while hopefully being useful for those future demands that we cannot anticipate. 
At the same time, it is important to acknowledge the limitations of what can be known to technology designers and they must be fundamentally open to all possible future use contexts. In other words, "one must take into account unintended uses and consequences, the constraints and potentials of materiality, and cultural contexts, which often are complex and multistable" (Ihde 2006, p. 51). With the approach we use in MultiVerse, we provide a mechanism for recording richer metadata such as intent and context, but the act of creating this data is itself associated with a source. This means that we are able to capture an author's original intent, but also are able to capture the intent assumed by any identifiable agent within the system. So to use the Thomas Edison example, the phonograph would be recorded with the information of how Edison intended it to be used, while allowing everyone who chose to use it in their own way to record their own intent should they wish (Ihde 2006; Lente 2006). The accuracy of intent can therefore be decided as a relative evaluation of the sources of the recorded intent, rather than argued as a single contentious property that needs to be resolved for a particular instance of the phonograph. This analogy is less metaphorical when we talk about literary works and their translations, for each translation represents the inferred intentions interpreted by the individual translators. Unless the original author provides a translation, there would be no equivalent to the Edison example. We hope this illustrates the importance of treating richer metadata as primary forms of data, with their own authorship and provenance for representing multicultural views. Such richer metadata may not address all possible problems, but offers a starting platform to do so.

\section{IIE and interpretive pluralism}

No news and information are merely factual but rather intrinsically embedded with ethical tones because people are influenced, as argued by Hongladarom, with "sets of values and justificatory systems" $(2009$, p. 2), originating from their differing cultures and traditions. As information technology greatly facilitates the movement of thoughts and ideas, they also increase chances of values clashing at an increasing rate, and raise the question of universality and distinctiveness of cultures. In this context, Intercultural Information Ethics (IIE) frameworks (Capurro 2008, 2009; Hongladarom and Britz 2010) can be an aid to discussing which set of values serve the existing goals, and fit with the desires of the people of a particular time and place, more than other values and, thereby, uphold ethical pluralism by recognizing the shared values and the irreducible differences between cultures and people. Ethical pluralism here needs to be differentiated from both ethical dogmatism and ethical relativism, because these approaches make each other untenable for the discussion of pluralistic views/values as supported by our MultiVerse. An ethical and epistemological relativism asserts that diversity and difference can only mean the lack of a single truth/value, while an ethical dogmatism insists on the homogenous application of a single ethical value and/or epistemological claim to truth-such that any different values, or truth claims, must be wrong/false (Ess 2006; Capurro 2008). Historically the concept of pluralism as something that posits multiple values came into existence with the gradual toleration of varying religious beliefs in the aftermath of religious wars in the sixteenth and seventeenth centuries, and then embracing respect of dissent and, finally, believing in the value of broader cultural diversity (Sartori 1997). Again pluralism can be of different types depending on the school of thought-e.g., differences between ancient western and eastern philosophical frameworks. Some examples of pluralism in the western philosophical traditions include Modus vivendi, liberal, complementarity, and interpretive (Ess 2006), while in the ancient eastern tradition would include the Confucian ren (authoritative humanity or co-humanity) and rites (Chan 2003, cited in Ess 2006, p. 219):

- Modus vivendi pluralism-recognizes "sheer differences between cultures and peoples" but presumes no existence of common ground between diverse cultures and views resulting in constant conflicts and confrontations (Ess 2006, p. 216).

- Robust pluralism-recognizes, based on Lawrence Hinman's idea of pluralism, compatibility in differing values and views without making the aspect of compatibility as a key requirement (Hinman, 1998, cited in Ess 2006, p. 216-217). In other words, robust pluralism emphasizes the fact that, "disagreement and difference are standard features of the moral landscape and can be sources of moral strength" (Hinman 2012, p. 45).

- Liberal pluralism-moves beyond the possibility of compatibility to various forms of connection and engagement between diverse ethical systems, based on John Rawls' (1993) notion of overlapping consensus, to find a shared set of ethical norms and standards for a shared existence (Madsen and Strong 2009, cited in Ess 2006, p. 217). But this insists on connection through identity.

- Complementarity pluralism-goes beyond a sheer identity of connections based on Charles Taylor's work on complementarity (2002) and John Rawls' work on overlapping consensus (1993) and traces a complementarity among differences to enrich a positive engagement between divergent parties/participants (Madsen and Strong 2009, cited in Ess 2006, p. 217). In other words, "complementarity relationships preserve and enhance the irreducible differences that define distinctive individuals, cultures, and civilizations" (Ess 2006, p. 217). 
- Interpretive pluralism-based on Plato's theory of ideas in The Republic (1974, Grube's translation) and Aristotle's pros hen ('towards one') equivocals in Metaphysics (1968, Tredennick's translation), interpretive pluralism is an acceptance of more than one ethical judgment regarding the interpretation and application of a shared ethical norm (cited in Ess 2006, p. 218). The pros hen equivocals demarcate a middle ground between homogeneous univocations (universalism - one and only one meaning) and pure equivocation (multiculturalism/multiplicitymultiple meanings entirely unrelated, sheerly different from one another).

- Confucian ren and rites-as Joseph Chan points out on the Confucian tradition, when the primacy of ren and rites is prevalent, Confucians are often ready to accept a plurality of diverse or even contradicting ethical judgments, as Confucius himself elaborates on this when he says, "If after careful and conscientious deliberation, two persons equipped with ren come up with two different or contradictory judgments and courses of action, Confucians would tell us to respect both of the judgments" (Analectus-Chan 2003, p.137, cited in Ess 2006, p. 219). This, of course, parallels the interpretive pluralism of Aristotle's notion of phronesis (practical judgment), and enables this ethical standard to be interpreted, applied, or understood in more than one way (Ess 2006).

Even though the main area of discussion and debate within IIE centers around the age-old philosophical problem of universalism and particularism, as Ess (2006), Capurro (2008, 2009), Hongladarom and Britz (2010), and others have argued, ethical pluralism is, and should be, at the core of IIE. Here, ethical pluralism, especially interpretive pluralism across cultures and traditions as discussed above, refers to the view that there are different, yet potentially equally legitimate ethical and cultural traditions in the world. As a result of recognizing the legitimacy of other ethical and cultural traditions, one cannot impose the normative standard of their own tradition upon people of other traditions without, at the same time, being guilty of ethical-cultural imperialism (Ess 2006). In other words, the openness of IIE allows multiple interpretations of norms and/or normative principles to be considered as valid, it also strips away one's ability to definitively determine which interpretation is superior. This interpretive pluralism is further allied with phronesis, the Aristotelean notion of practical judgment. Such practical judgment thus allows one to draw distinctively different ethical conclusions applying shared principles to diverse contexts (Ess 2006). In contrast with simple but only potential compatibility (Hinman's robust pluralism) and connection by way of sheer identity (Madsen and Strong's liberal pluralism), this form of pros hen, interpretive pluralism, is inextricably tied to the central facility of judgment, and thus allows for a strong form of connection that preserves irreducible differences.

Here we adapt the IIE framework with interpretive pluralism and also Confucian ren and rites, at its core to discuss the pluralistic views and values captured and connected by our MultiVerse prototype. MultiVerse is deliberately designed to exploit structures of connection alongside irreducible differences, and to preserve them not only for later retrieval, but also to help interpret, apply, or understand in more than one way. For example, MultiVerse both facilitates and expresses this interconnectedness through its maintenance of provenance chains and additional richer metadata. In other words, in addition to the preservation of multiple versions and variations of a particular text or data file, the provenance (the path to its creation, and the sources upon which it builds) are also maintained. Files and texts are therefore preserved specifically as structures of unitary foci that include diverse, even contradictory interpretations (and not simply as a repository of multiple forms). For example, a particular verse of a translated copy of Dante's Divine Comedy could be recorded by a MultiVerse user, then could be further annotated either by the same user, or other users, to complement the text by adding nuances upon, or even negate with contradictory views of the text (or a particular version of it). Unlike a simple multi-variate repository of data (Modus vivendi pluralism or Liberal pluralism), the preservation of the identities and paths of interpretation, and the context (e.g., the specific translation used), all contribute to a much richer view of the individual editors' and annotators' contributions. MultiVerse achieves this goal by decoupling itself from the task of judging the 'truthness' or superiority of one narrative over the other. All narratives and their interpretations are recorded and, when required, can be complemented and compared as shown in Fig. 3 in line with the IIE framework. Thus, the MultiVerse offers a distinctive strength by design, that it can move beyond tracing sheer differences of modus vivendi pluralism (Ess 2006) between various narratives, beyond minimal compatibility of robust pluralism, and beyond an instance of connection by sheer identity of liberal pluralism by developing instead relations of complementarity that preserves the irreducible differences defining the Other as Other which is the core aspect of the interpretive pluralism of IIE and Confucian ren and rites. Thus, MultiVerse avoids homogenizing differences in the name of an overriding shared identity and provides an infrastructure for interpretive pluralism, that Capurro, Ess, Hongladarom, and Britz (among others) defend in IIE. 


\section{Poststructuralism-hypertext/hypermedia}

In this section, we briefly discuss the relevance of MultiVerse in the context of poststructuralism, literary theory, and hypertext/hypermedia. ${ }^{3}$ To do this, it evidently warrants at least a cursory discussion of poststructuralists/hypertexttheorists' concepts of intertextuality, multivocality, decentering, and multilinearity and their relation to MultiVerse.

Structuralism argues that social structures are themselves objects or events that contain meanings or signs (Tredinnick, 2007), and when it is extended to media technologies it tends to interpret media text as having only one possible interpretation or one absolute meaning. As noted in (Tredinnick 2007, p. 173-174), Ferdinand de Saussure (1966), a structuralist, explains signs in two parts: as the 'signifier' meaning the text, image, sound, or object, and the 'signified' meaning the concept the signifier represents. Meanings are generated/understood in relation to the signifier and the signified. For example, a signifier 'cat' should always refer to a signified felid in general and specifically felis catus - domestic cat, not something else. A closer look at this mechanism, as observed by Chandler (2007), reveals that truth seeking is nothing more than looking at reality as unequivocal through oppositions or binary pairs such as, for the given example, tree versus shrub or tree versus animal. Furthermore, as de Saussure asserted, all signs or meanings are constructed culturally and ideologically mediated through mass media texts whereby a society's ideological interpretations of media texts solely become universal reflections of everyday life (de Saussure 1966, cited in Tredinnick 2007, p. 174).

In contrast to structuralists' ideas of singular universal reality, poststructuralists, such as Jacques Derrida, Michel Foucault, and Roland Barthes, stress the plurality of signification by focusing less on the structure of text, but more on the role of a reader (audience) as interpreter, in the production of meaning (Landow 2006). For example, Derrida (1978), proposing his deconstruction theory, argues that a text is itself an endless stream of signifiers and consists of multiple interpretations in which true meaning is impossible to pin down. Similarly Barthes, using literary theory, introduces 'readerly text' and 'writerly text' to explore the nature of a text as a mixture of texts drawn from numerous writings or traditions and the meaning of a text resides in the way readers consume it, because the text is specifically written for them (Barthes 1977). For Barthes, writerly text places readers as active constructors of meaning(s), while readerly text assumes passive readers focused on the text's one meaning. In a similar vein,

\footnotetext{
${ }^{3}$ Hypermedia simply extends the notion of the text in hypertext by including visual information, sound, animation, and other forms of data. Both terms refer to a system of linked information. In this paper, we therefore use the terms hypertext and hypermedia interchangeably.
}

Foucault insists on the multiplicity of meanings of a text, when he acknowledges the role of 'author function' in which an author only sets a part of a written work to aid in regularizing discourse and knowledge, but not the entire interpretative process by readers (Foucault 1997).

Interestingly, the poststructuralists' notions of text can now be tested in one of the computer writing techniques known as computer hypertext in which poststructuralism conceives of text in terms of network and links (Landow 2006). Literary theory and computer hypertext, though unconnected areas of inquiry, have increasingly converged, to bring about a paradigm shift in the reading/writing of literary works and in web development by changing the world's way of seeing, accessing, and connecting information through the lens of poststructuralism. Hypertextuality embodies poststructuralist conceptions of the 'open text'text composed of blocks of text (lexia-a poststructuralists' term) — and the electronic links that join them, that may be described by terms like link, node, network, web, and path. That is to say, electronic links connect lexias "external" to a work-a commentary on it by another, or parallel, or contrasting texts, as well as within it, and thereby create text that is experienced as nonlinear, or multilinear (Landow 2006). Although conventional reading habits apply within each lexia, once one leaves the shadowy bounds of any text unit, new rules and new experience apply. This is what Thomas H. Nelson who coined the term 'hypertext' in the 1960s, at a time when the idea of using computers for media was radical, defines it as non-sequential writing read at interactive screen "to mean a body of written or pictorial material interconnected in such a complex way that it could not conveniently be presented or represented on paper" (Nelson 1965, p. 96). As popularly conceived, this is "a series of text chunks connected by links which offer the reader different pathways" (Landow 2006, p. 3). Of the parallels between critical theory and computer hypertext, the most important thing according to Landow is the fact that "critical theory promises to theorize hypertext and hypertext promises to embody and thereby test aspects of theory, particularly those concerning textuality, narrative, and roles or functions of reader and writer" (Landow 2006, p. 52). In particular, poststructuralists view text in terms of network and links that have several implications (Foucault 1997; Landow 2006; Barthes 1997). What follows are some of the key concepts of text in poststructuralist thinking, such as intertextuality, multivocality, decentering, and multilinearity, that represent the way interactive narratives work in hypertexts, and their relation to MultiVerse.

\section{Intertextuality}

The notion of intertextuality is a mixture of other writings becoming, as Barthes describes, "a multidimensional space 
in which a variety of writings, none of them original, blend, and clash" (Barthes 1977, p. 151). From this perspective, a text does not convey a single narrative strand with a particular author's meaning, but rather a combination of several voices from different authors, and a compilation of cultural textuality. Intertextuality has its root in print technology. For example, footnotes or quotations that are indicative of other source texts outside the main text. However, the concept of intertextuality is stronger in hypertext than its printed one. In hypertext, intertextuality is manifested through hyperlinks whereby other texts from different authors can be brought together in a single node. As Allen (2000) points out, hypertext systems allow us to take on a trajectory moving from the main text into multiple intertextual pathways even to the extent that the main text may seem just one among the many texts in the hypertext chain. In fact, this intertextuality is achieved through the preservation of multiple voices and their interlinks are retrieved through the provenance chain in the MultiVerse. For instance, when a text is selected in MultiVerse, it can show its interdependency by highlighting how this selected text stands in relation to another and produces meaning/connectivity, as shown in Fig. 3. This feature, in the design and development of MultiVerse, can in turn can serve to support multiculturality through the preservation of this added meaning as an aid to more accurate and open translations and annotations. Therefore, hypertext readers can open up their reading to an apparently boundless play of relationships through intertextuality without limiting it to an author and literary tradition alone.

\section{Multivocality}

The term multivocality is closely related to intertextuality. For Barthes (1977), however, a text is plural in meaning, not simply by virtue of having several meanings, but in terms of achieving the 'very plural meaning' involving the play of signifiers. It is this 'very plural meaning' that brings forth the idea of multivocality. A text is multivocal in the sense that it co-joins multiple voices drawn from several discourses with each having equal expression. Landow (2006) asserts that hyperlinks and multilinear means of structuring narrative enable hypertext as multivocal. Textual representations can be easily composed and compared from different positions or voices. In this way, texts are no longer bound by the physical limitations of print which fosters the primacy of a univocal voice. In MultiVerse, by design, users have a greater role and freedom to record and associate a set of events/texts (i.e., combine and remix verses to form multiple versions of a canto in each canticle, with relevant annotations of context and sentiments, thereby generating multiple new versions of the 'Divine Comedy,' as shown in Fig. 2) and possibly gain aesthetic pleasure by such an interactivity. Therefore, by playing within diverse perspectives enabled by MultiVerse, users can organize their own stories/events/ texts and not only support the recording of existing plural meanings, but create new texts that receive a very plural meaning of their own.

\section{Decentering}

Derrida (1978) argues, in his theory of deconstruction, that all structure, including language, contains some sort of 'centre' the function of which "was not only to orient, balance, and organize the structure ... but above all to make sure that the organizing principle of the structure would limit what we might call the free play of the structure" (p. 278). Even though Derrida acknowledged that the center is important, he emphasized that meaning is not fixed. That is to say that what the center determines cannot represent a final signified because it can be a signifier itself. As a result, a chain of signifiers with the center continuously shifting between the signified and signifier, the center becomes decenteredwhich is the idea of decentering. This provides freedom and flexibility to traverse from the centre to the periphery and vice versa as one deems fit. In MultiVerse, decentering is experienced through the parallelisms between competing voices and their dynamic interactions expressed through their provenance chains. Therefore, the characteristic of MultiVerse is said to manifest this kind of decentering experience in the narrative because it enables users/readers, using the provenance chain of a text, to move from one text to another with each representing a potential new center and focus of their investigation.

\section{Multilinearity}

In literary writing with a sequence of paragraphs with specific functions, a linear structure is organized to reflect a writer's knowledge and/or arguments about a subject as well as to direct a reader's experience. Hypertext, in contrast, is a non-linear with no specific center because it is composed of bodies of linked text that have no primary axis of organization, resulting in a multilinearity of texts, i.e., networks of textual data. Hypertext can be challenging as readers may have to continually shift their attention each time they come across a new text. This could be a benefit in the sense that readers gain freedom to explore various paths for exploration and to determine their center of investigation without any restriction from the author. Some readers may find reading hypertext enjoyable. However, readers who are more oriented to a single narrative structure may find it difficult to stay focused. With the lack of a fixed center to guide reading, readers can also be confused about where to go next in the electronic space. The experience can be discouraging because readers feel trapped and lost within the vast hypermedia system. Kim and Stephen (1995) explain 
that this loss of one's sense of direction, position, or relationship with one's surroundings, is disorientation. Similarly, in MultiVerse, users can be distracted by multiple narrative threads that can be made available to them through references, annotations, and provenance chains. But hypertext theorists believe that the thematic coherence between oral and visual languages may provide reading choices that may encourage readers to explore the content of each option (Kim and Stephen 1995). Each of these choices could provide an element of surprise, or even disappointment, to readers. In MultiVerse, there is a potential for multilinearity, but this is not really an inherent problem of the design of MultiVerse as a data creation and preservation scheme, but is rather a question of data and system presentation, and its suitability to a particular audience.

The design of MultiVerse attempts to bring together different poststructural concepts, media capability, and interpretive traditions to produce entirely distinct and artistic literary works which are pluralistic and multicultural in nature, by providing technological support and control/freedom to creators and scholars in choosing/authoring their own paths, and in recording their interpretations and meanings. When authoring in MultiVerse, users as authors have more liberty in the textual selections through electronic links. With the necessity to choose links that do not always move linearly, the user takes on a more active role in deciding their own authoring, as well as reading, directions. Because every text which is inserted in MultiVerse creates a web of relations and allows non-sequential reading, authoring, and perhaps even thinking. As a result, users as readers may encounter many other texts/comments/annotations that are created by different users/authors. Instead of reading a text which creates the illusion that it is constructed by a single author, or has had only one path to its current form, the text now includes many individuals who are associative of each other in the text creation. Apart from the liberty of choosing a reading direction from the links, authors and readers can make changes to an existing document or create annotations, not by deleting them and replacing them with new texts, but by editing new versions and adding annotations to the existing ones, so that nothing is lost once created and recorded. This immutability is achieved through the implementation of an immutable storage system, leveraging a form of blockchain technology, as part of the MultiVerse design model. Therefore, users can incorporate other texts into a document through electronic links, creating a corpus of interlinked texts/materials. Such liberty given by electronic links has several implications. For example, as noted by Landow and Delany (1991), the electronic linking shifts the boundaries between individual works and those between author and reader and engenders the notion of "authorial property, authorial uniqueness, and a physically isolated text of print" (p. 7).

\section{Data privacy and other questions}

We argue, as discussed above (under "Digital hermenuetics and contextualism" and "Multistability and multiculturality"), that the epistemic responsibility in a multicultural setting is easier to meet when the underlying information infrastructure does not impose an unavoidable loss of information through adaptation/translation/use. In present formats, the existing published works do not provide for a means to capture broader contexts, making it such that deciphering the author's intended meanings, or the origins of the meanings we assume to be the author's, become more complex and tenuous. In contrast, MultiVerse eases this process by avoiding default interpretations entirely, and providing a flexible data representation that can accommodate multiple alternatives of texts and interpretations. Moreover, it helps to present alternatives to how we think of an object (technology), not the nature of things as they are, but how we want to represent them with embedded richer-referential metadata, subtle nuances, and underlying contexts. Another interesting feature in MultiVerse is the ability to share the history of paths of a digital product to compare and contrast, for example an art work, either as an end result or as a dynamic creative process of creating it.

However, one might perceive MultiVerse as a system that is deliberately designed to record too much metadata, thereby creating an unnecessary information overload and data privacy; or as a scientific apparatus to dissect the intellectual work of others; or as a blockchain mechanism to prevent the ability to edit what is stored. To prevent these undesired consequences, there is a choice, by design, for users either to opt out from recording all their creative activities or to opt in to reveal as much as it is needed or to choose documenting the synthesizing process of a digital product.

Concerning the question of data privacy which, while orthogonal to the proposed structure and use of the system, is nonetheless an important issue in any specific deployment. Determining how the data is gathered, and who has access to it is not particularly problematic when the intent is the collection and dissemination of published works, since the assumption is that publication implies a desire for broader access. Issues of access control (e.g. due to systems that require payment, or which place content behind a firewall) are not particularly relevant when MultiVerse essentially needs to hold little more than a pointer to the source in order to serve its function. Where this issue needs greater care and refinement in future work is in controlling the ability to update and add to a MultiVerse archive.

On further reflection, these ethical questions raise other legitimate concerns like the interpretation of novelty, the contribution of authorship, the responsibility of attribution, and the claim of intellectual property, and the very independent legitimacy of the thing itself. These are difficult 
questions, but an inability to record enough data to pose them, is no answer. The fact that our proposed infrastructure raises the complexity of questions discussed, is a boon.

\section{Applications of MultiVerse in broader domains}

The idea that the digital infrastructure needs to be multicultural could benefit many domains, not just the content/ people/policies that engage with such infrastructure. Some examples could include: legal databases, interdisciplinary dictionaries and encyclopedias, better tracking and sharing of scientific scholarship, Biblical hermeneutics, and less contentious preservation of scriptural texts that have delicate and critical provenance considerations.MultiVerse, reviving the promise of hypertext in the early days of the Internet, is better suited for hermeneutics of documents in which references to other parts of the document, or to other documents, is a part of its structure, e.g., the Talmud. ${ }^{4}$ The Talmud, with its heavy use of annotations and nested commentary and its reliance on references to other sources, is in fact portrayed as an ancient prototype of hypertext (Conklin 1987; Porush 1998; Davidson 2011). The primordial hypertextual structure of Talmud, as well as in most other forms of modern day hypertext/hypermedia, is echoed and extended in MultiVerse. Within the domain of other scriptural texts and commentaries, imagine the Pentateuch ${ }^{5}$ tagged with provenance and author tags, or two competing interpretations of a scriptural verse being accessible without contention. The flipped perspective of anticipated use of technology design can make it unnecessary to highlight the different sources until desired. For the Pentateuch example, such an approach would make it easy to produce a more dynamic document, in the vein of The Bible with sources revealed (Friedman 2009). Or imagine running the two major Sanskrit epics of

\footnotetext{
4 Talmud: A primary source of Jewish religious tradition, law, custom, and culture. The Talmud consists of what are known as the Gemara and the Mishnah, infused with vigorous intellectual debate, humor, and deep wisdom.

5 Pentateuch: It refers to the first five books of the Bible. These five books are Genesis, Exodus, Leviticus, Numbers, and Deuteronomy. This is attributed to the Israelite leader, Moses.

${ }^{6}$ Ramayana: It is an ancient Indian epic on the life of Rama, a legendary prince of the Kosala Kingdom. This is attributed to Maharishi Valmiki.

${ }^{7}$ Mahābhārata: Mahābhārata is another ancient Indian epic about the battle between two princely families - the Pandavas and Kauravas - in the Kurukshetra war for their royal succession. This is attributed to Vyasa.

8 Sahīh/Hadith: a collection of traditions containing sayings of the prophet Muhammad. One of the best known Șahịh is attributed to Muhammad al-Bukhari.
}

ancient India: the Ramayana ${ }^{6}$ or the Mahäbhärata ${ }^{7}$ through a linguistic/regional predictor to be better able to map the geography of the individual tales that formed these sweeping and masterful epics. Or imagine the Sahī $h^{8}$ of Muhammad al-Bukhari (Khan 1986), a recording of the Islamic prophetic traditions (known as the Hadith), with its complied and codified provenance information more accessibly tagged for easier scholarly exegesis, and eliminating contention regarding conflicting interpretations.

All these are examples of how an approach to data representation like MultiVerse can be not simply an improved approach to supporting the preservation of data and the contexts that generated it. But rather that such an ability is particularly useful, and a direct benefit, for cases where information is lost when it is adapted across cultures and individuals.

\section{Conclusion}

In this paper, we explored a novel idea of rethinking how we capture richer metadata and representing the same accurately, by technology design from the beginning, can impact positively users' technology adaptation, use, and multicultural perspectives. We demonstrated this idea through an implementation of a digital infrastructure, MultiVerse. This digital multicultural framework provided a convenient medium for users to interact with existing literary sources as well as to generate new derivatives thereof with ease with options of choosing a degree of complexity. Furthermore, the system ensured a holistic preservation of provenance metadata of users' technology interactions without losing meaning of their intentions and contexts with an additional feature to attribute credits to their creative works. We also examined several ethical implications of MultiVerse through multiple lenses including digital hermeneutics perspectives, IIE, and poststructuralism, and evaluated the feasibility of the proposed framework in broader domains. We hope that the success and maturity of MultiVerse as a digital infrastructure prototype for multicultural perspectives will make the notion of anticipating intercultural pluralistic views at the very initial technology design phase, a common practice, rather than as an after-thought consideration (and be implemented in broader digital humanities and humanities fields).

Open Access This article is licensed under a Creative Commons Attribution 4.0 International License, which permits use, sharing, adaptation, distribution and reproduction in any medium or format, as long as you give appropriate credit to the original author(s) and the source, provide a link to the Creative Commons licence, and indicate if changes were made. The images or other third party material in this article are included in the article's Creative Commons licence, unless indicated otherwise in a credit line to the material. If material is not included in 
the article's Creative Commons licence and your intended use is not permitted by statutory regulation or exceeds the permitted use, you will need to obtain permission directly from the copyright holder. To view a copy of this licence, visit http://creativecommons.org/licenses/by/4.0/.

\section{References}

Albrechtslund, A. (2007). Ethics and technology design. Ethics and information technology, 9(1), 63-72.

Allen, G. (2000). Intertextuality. New York, NY: Routledge.

Archival Education and Research Institute (AERI) and Pluralizing the Archival Curriculum Group (PACG). (2011). Educating for the Archival Multiverse. The American Archivist, 74, 69-102.

Ashby, S., Hanna, J., Matos, S., Nash, C., \& Faria, A. (2019). FourthWave HCI Meets the 21st Century Manifesto. Proceedings of the Halfway to the Future Symposium, 2019, 1-11.

Barad, K. (2007). Meeting the Universe Halfway: Quantum Physics and the Entanglement of Matter and Meaning. Durham: Duke University Press.

Barthes, R. (1977). Image-music-text. In S. Health (Ed.), Trans (Vol. 332). London, England: Fontana Press.

Beyer, H., \& Holtzblatt, K. (1999). Contextual design. interactions, $6(1), 32-42$.

Bødker, S. (2006). When second wave HCI meets third wave challenges. In Proceedings of the 4th Nordic conference on Humancomputer interaction: changing roles, 1-8.

Bødker, S. (2015). Third-wave HCI, 10 years later-participation and sharing. Interactions, 22(5), 24-31.

Botsman, R. (2017). Big data meets Big Brother as China moves to rate its citizens. Wired $U K, 21$. Available at https://www.wired.co.uk/ article/chinese-government-social-credit-score-privacy-invasion

Brigham, M., \& Introna, L. (2007). Invoking politics and ethics in the design of information technology: Undesigning the design. Ethics and Information Technology, 9(1), 1-10.

Butterfield, A. D. (2012). Ethnographic assessment of quantified self meetup groups. Unpublished Doctoral dissertation, San José State University.

Cappelen, H., \& Lepore, E. (2005). Insensitive Semantics. Oxford: Blackwell.

Capurro, R. (2008). Intercultural information ethics: foundations and applications. Journal of Information, Communication and Ethics in Society, 6(2), 116-126. https://doi.org/10.1108/1477996081 0888347.

Capurro, R. (2009). Intercultural information ethics: foundations and applications. Signo Y Pensamiento, 28(55), 66-79.

Capurro, R. (2010). Digital hermeneutics: an outline. AI \& society, 25(1), 35-42.

Carr, B., \& Ellis, G. (2008). Universe or multiverse? Astronomy \& Geophysics, 49(2), 2-29.

Chan, J. (2003). Confucian Attitudes Towards Ethical Pluralism. In R. Madsen \& T. B. Strong (Eds.), The Many and the One: Religious and Secular Perspectives on Ethical Pluralism in the Modern World (pp. 129-153). Princeton: Princeton University Press.

Chandler, D. (2007). Semiotics: The basics. New York, NY: Routledge.

Chilana, P. K., Ko, A. J., \& Wobbrock, J. (2015). From user-centered to adoption-centered design: A case study of an HCI research innovation becoming a product. In Proceedings of the 33rd Annual ACM Conference on Human Factors in Computing Systems, 1749-1758.

Cohen, S. (1999). Contextualism, skepticism, and the structure of reasons. Philosophical perspectives, 13(1), 57-89.

Collingridge, D. (1980). The Social Control of Technology. United Kingdom: Frances Pinter.
Conklin, J. (1987). Hypertext: an introduction and Survey. Computer, 20(9), 17-41.

Creemers, R. (2018). China's Social Credit System: An Evolving Practice of Control. SSRN scholarly paper ID SSRN 3175792. Rochester: Social Science Research Network.

Cronin, M. (2012). Translation in the digital age. New York: Routledge.

Davidson, J. (2011). Paths and Pointers: Remediation and/as Talmudic commentary in a video clip on KosherTube. Student Journal of Canadian Jewish Studies, 1-30.

de Saussure, F. (1966), Course in General Linguistics, Bally, C. and Sechehaye, A. (Eds.), in collaboration with Riedlinger, A., translated with an Introduction by Baskin, W., New York: McGraw-Hill.

Derrida, J. (1978). Structure, sign, and play in the discourse of the human sciences. In L. Hutcheon (Ed.), A postmodern reader (pp. 278-294). New York: State University of New York Press.

Deutsch, D. (2002). The structure of the multiverse. Proceedings of the Royal Society of London. Series A: Mathematical, Physical and Engineering Sciences, 458(2028), 2911-2923.

Endsley, M. R. (2016). Designing for situation awareness: An approach to user-centered design. Boca Raton: CRC Press.

Ess, C. (2006). Ethical pluralism and global information ethics. Ethics and Information Technology, 8(4), 215-226.

Fernaeus, Y., Ljungblad, S., Jacobsson, M., \& Taylor, A. (2009). Where third wave HCI meets HRI: report from a workshop on user-centred design of robots. In 2009 4th ACM/IEEE International Conference on Human-Robot Interaction (HRI), 293-294.

Foucault, M. (1997). What is an Author? In D. F. Bouchard \& S. Simon (Eds.), Language, Counter-Memory Practice: Selected Essays and Interviews (pp. 113-138). Ithaca, New York: Cornell University Press.

Friedman, B., Kahn, P. H., \& Borning, A. (2002). Value Sensitive Design: Theory and Methods. University of Washington Technical Report, 2-12.

Friedman, B., Kahn, P. H., \& Borning, A. (2006). Value Sensitive Design and Information Systems. In P. Zhang \& D. Galletta (Eds.), Human-Computer Interaction and Management Information Systems: Foundations. M.E. Sharpe: Armonk.

Friedman, R. E. (2009). The Bible with sources revealed. New York: Harper Collins

Gallivan, M., \& Srite, M. (2005). Information technology and culture: Identifying fragmentary and holistic perspectives of culture. Information and organization, 15(4), 295-338.

Gilliland, A. J., \& Willer, M. (2014). Metadata for the information multiverse. In iConference 2014 Proceedings, 1117-1120. doi:https://doi.org/10.9776/14396

Gimpel, H., Nißen, M., \& Görlitz, R. (2013). Quantifying the quantified self: A study on the motivations of patients to track their own health. In Proceedings of the Thirty Fourth International Conference on Healthcare Information Systems, Milan. 1-16.

Grube, G. M. A., \& (Trans.). . (1974). Plato'sThe Republic. Massachusetts: Hackett Publishing.

Helander, M. G. (Ed.). (2014). Handbook of human-computer interaction. New York: Elsevier.

Hinman, L. M. (1998). Ethics: A pluralistic approach to moral theory. Fort Worth, Harcourt, Brace: Nelson Education.

Hinman, L. M. (2012). Ethics: A Pluralistic Approach to Moral theory, 5th International Edition. Clark Baxter: Cengage.

Ho, D. Y. (1993). Relational orientation in Asian social psychology. In U. Kim \& J. W. Beny (Eds.), Indigenous psychologies: Research and experience in cultural context (pp. 240-259). Newbury Park, CA: Sage.

Ho, D. Y. (1995). Selfhood and identity in Confucianism, Taoism, Buddhism, and Hinduism: contrasts with the West. Journal for the theory of social behaviour, 25(2), 115-139. 
Hoffman, S. R. (2017). Programming China: The Communist Party's autonomic approach to managing state security (Doctoral dissertation), University of Nottingham.

Holtzblatt, K. (2009). Contextual design. In A. Sears \& J. A. Jacko (Eds.), Human-Computer Interaction (pp. 56-70). Boca Raton: CRC Press.

Hongladarom, S., \& Britz, J. (2010). Intercultural information ethics. The International Review of Information Ethics, 13(1), 2-5.

Ihde, D. (1999). Technology and prognostic predicaments. AI \& Society, 13(1), 44-51.

Ihde, D. (2004). Philosophy of Technology. In P. Kemp (Ed.), Philosophical Problems Today. Philosophical Problems Today (Vol. 3, pp. 91-108). Dordrecht: Springer.

Ihde, D. (2006). The Designer Fallacy and Technological Imagination. In J. R. Dakers (Ed.), Defining Technological Literacy (pp. 121-131). New York: Palgrave Macmillan.

Ihde, D. (2012). Technics and praxis: A philosophy of technology (Vol. 24). Netherland: Springer.

Karray, F., Alemzadeh, M., Saleh, J. A., \& Arab, M. N. (2008). Human-computer interaction: Overview on state of the art. International Journal on Smart Sensing and Intelligent Systems, 1(1), 137-159.

Kelly, M. (2016). Hermeneutics and Information Science: The Ongoing Journey From Simple Objective Interpretation to Understanding Data as a Form of Disclosure. In M. Kelly \& J. Bielby (Eds.), Information Cultures in the Digital Age (pp. 83-110). Wiesbaden: Springer VS.

Khan, M. M. (1986). Sahih Bukhari. Islamabad: Peace Vision.

Kim, H., \& Stephen, C. H. (1995). Spatial metaphors and disorientation in hypertext browsing. Behaviour \& Information Technology, 14, 239-250.

Kostka, G. (2019). China's social credit systems and public opinion: Explaining high levels of approval. New media \& society, 21(7), $1565-1593$.

Kudina, O., \& Verbeek, P. P. (2019). Ethics from within: Google Glass, the Collingridge dilemma, and the mediated value of privacy. Science, Technology, \& Human Values, 44(2), 291-314.

Kushniruk, A., \& Nøhr, C. (2016). Participatory design, user involvement and health IT evaluation. Stud Health Technol Inform, 222, $139-151$.

Kyng, M., \& Greenbaum, J. (1991). Cooperative design: bringing together the practices of users and designers. In IFIP TC8/WG 8.2 Working Conference on the Information Systems Research Arena of the 90's (pp. 405-416). North-Holland.

Landow, G. P. (2006). Hypertext 3.0: Critical theory and new media in an era of globalization. Baltimore, MD: JHU Press.

Landow, G. P., \& Delany, P. (1991). Hypertext, hypermedia and literary studies: The state of the art. In P. Delany \& G. P. Landow (Eds.), Hypermedia and literary studies (pp. 3-50). Massachusetts, MA: MIT Press.

Lanzing, M. (2019). "Strongly recommended" revisiting decisional privacy to judge hyper nudging in self-tracking technologies. Philosophy \& Technology, 32(3), 549-568.

Leidner, D. E. (2006). A review of culture in information systems research: Toward a theory of information technology culture conflict. MIS Quarterly, 30(2), 357-399.

Lente, H. (2006). Expected behavior: Anticipation of use in technological development. In P.-P. Verbeek \& A. Slob (Eds.), User behavior and technology development: Shaping sustainable relations between consumers and technologies. Eco-Efficiency in Industry and Science (Vol. 20, No. 1, pp. 211-219). Springer.

Liang, F., Das, V., Kostyuk, N., \& Hussain, M. M. (2018). Constructing a data-driven society: China's social credit system as a state surveillance infrastructure. Policy \& Internet, 10(4), 415-453.
Madsen, R., \& Strong, T. B. (2009). The many and the one: Religious and secular perspectives on ethical pluralism in the modern world. New Jersey: Princeton University Press.

Mehrez, S. (2012). Introduction: Translating revolution: An open text. In S. Mehrez (Ed.), Translating Egypt's revolution: The language of Tahrir (pp. 1-24). Cairo: AUC Press.

Nelson, T. H. (1965). Complex information processing: a file structure for the complex, the changing and the indeterminate. In Proceedings of the 1965 20th national conference (pp. 84-100).

Orgad, L., \& Reijers, W. (2019). A Dystopian Future? The Rise of Social Credit Systems. Robert Schuman Centre for Advanced Studies Research Paper No. RSCAS, 94.

Porush, D. (1998). Talmud as Hypertext, In 'Contra', KAIROS, 3(1).

Preece, J., Rogers, Y., Sharp, H., Benyon, D., Holland, S., \& Carey, T. (1994). Human-computer interaction. Addison-Wesley Longman Ltd.

Rawls, J. (1993). Political Liberalism. New York: Columbia University Press.

Romele, A., Severo, M., \& Furia, P. (2020). Digital hermeneutics: from interpreting with machines to interpretational machines. AI \& SOCIETY, 35, 73-86. https://doi.org/10.1007/s00146-018-0856-2.

Salvo, M. J. (2001). Ethics of engagement: User-centered design and rhetorical methodology. Technical communication quarterly, 10(3), 273-290.

Sartori, G. (1997). Understanding pluralism. Journal of democracy, $8(4), 58-69$.

Scanlon, T. (2000). What we owe to each other. Cambridge: Harvard University Press.

Schuler, D., \& Namioka, A. (Eds.). (1993). Participatory design: Principles and practices. Boca Raton: CRC Press.

Soothill, W. E. (1910). The analects of Confucius. Shansi: T'aiyuanfu.

Spinuzzi, C. (2005). The methodology of participatory design. Technical communication, 52(2), 163-174.

Stine, G. C. (1976). Skepticism, relevant alternatives, and deductive closure. Philosophical Studies, 29(4), 249-261.

Suchman, L. (2007). Human-Machine Reconfigurations: Plans and Situated Actions (2nd ed.). Cambridge: Cambridge University Press.

Swierstra, T., Stemerding, D., \& Boenink, M. (2009). Exploring Techno-Moral Change: The Case of the ObesityPill. In P. Sollie \& M. Düwell (Eds.), Evaluating New Technologies (Vol. 3, pp. 119-138). Springer, Dordrecht: The International Library of Ethics Law and Technology.

Taylor, C. (2002). Modern social imaginaries. Public culture, 14(1), 91-124.

Te'eni, D. . (2006). Designs that fit: an overview of fit conceptualization in HCI. In P. Zhang \& D. Galletta (Eds.), Human-Computer Interaction and Management Information Systems: Foundations, 5 (pp. 61-89). Armonk: M.E. Sharpe.

The Dartmouth Dante Project (DDP). (2013). Multiple translations of Divine Comedy. Available at http://dantelab.dartmouth.edu/reader

Tredennick, H., \& (Trans.), . (1968). Aristotle's Metaphysics. Cambridge, Mass: Harvard University Press.

Tredinnick, L. (2007). Post-structuralism, hypertext, and the World Wide Web. Aslib Proceedings of New Information Perspectives, 59(2), 169-186

Tripathi A.K. (2016) The Significance of Digital Hermeneutics for the Philosophy of Technology. In M. Kelly \& J. Bielby (Eds.), Information Cultures in the Digital Age. (pp. 143-157). Springer VS, Wiesbaden. https://doi.org/https://doi.org/10.1007/ 978-3-658-14681-8_8

van de Poel, I. (2013). Why new technologies should be conceived as social experiments. Ethics, Policy \& Environment, 16(3), 352-355.

van der Velden, M. (2009). Another design is Possible: Looking for ethical agency in global information and communication technology. (PhD dissertation). Bergen: University of Bergen. 
van der Velden, M. (2010). Undesigning Culture: A brief reflection on design as ethical practice. In F. Sudweeks, H. Hrachovec, \& C. Ess (Eds.), Proceedings Cultural Attitudes Towards Communication and Technology (pp. 117-123). Australia: Murdoch University.

Watson, G. (2002). Contractualism and the Boundaries of Morality: Remarks on Scanlon's What We Owe to Each Other. Social Theory and Practice, 28(2), 221-241.

Watson, R. T., Ho, T. H., \& Raman, K. S. (1994). Culture: A fourth dimension of group support systems research. Communications of the ACM, 37(10), 44-56.
Wong, K. L. X., \& Dobson, A. S. (2019). We're just data: Exploring China's social credit system in relation to digital platform ratings cultures in Westernized democracies. Global Media and China, $4(2), 220-232$.

Yeung, K. (2017). 'Hypernudge': Big Data as a mode of regulation by design. Information, Communication \& Society, 20(1), 118-136.

Publisher's Note Springer Nature remains neutral with regard to jurisdictional claims in published maps and institutional affiliations. 